\title{
Current Challenges in the Application of Agent Theory to Construction Management in Innovative, Intelligent Field Factory
}

\author{
Shigeomi Nishigaki ${ }^{1}$ \\ Masanori Kaku ${ }^{2}$ \\ Yoshio Maruyama $^{3}$ \\ Kinya Tamaki ${ }^{4}$
}

\begin{abstract}
This research project is a part of IMS IF7 titled "Innovative, Intelligent Field Factory, " which is one of IMS research projects in Japan. The research program of IF7 consists of the three work packages. In work package 1, particular emphasis will be placed on realization of modularized construction system. Work package 2 includes fundamental studies on automated construction systems. In work package 3, studied is the application of agent theory to construction management. The work package 3 has the three tasks. Task 1 is to develop virtual construction dynamic simulation systems. In task 2, studied is field factory transparency where detailed information, proactive advises and guidelines in all locations are accessible electronically by workers for thinking and decision-making. Task 3 is to apply agent theory to safety management, resources management, facility management and large-scale distributed information management. This paper presents on-going research activities of the working package 3 .
\end{abstract}

\section{1: Introduction}

This research project is a part of IMS IF7 titled "Innovative, Intelligent Field Factory," which is one of IMS research projects in Japan. Intelligent Manufacturing System (IMS) program was proposed by Japan in 1989. The objectives of IMS program are to provide an exciting opportunity for international cooperation in advanced manufacturing, and a vision and structure for world-wide sharing of manufacturing technology development with costs, risks and benefits in a balanced and equitable manner. The major thrust areas are of product life cycle issues, process issues, strategy, planning and design tools, human, organization and social issues, and virtual extended enterprise issues. Three committees, international steering committee, technical committee and intellectual property right committee are appointed by the United States, Canada, EU, Australia and Japan.

The research program of IF7 consists of the three work packages as follows.

\section{Work package 1:}

Fundamental studies on new advanced construction for large-scale 3D structures: Within this work package, particular emphasis will be placed on realization of modularized construction system by utilizing flexible advanced manufacturing technologies.

\footnotetext{
${ }^{1}$ Senior Research Engineer, Technical Research Institute, Hazama Corp., 515-1 Nishi-mukai Karima, Tsukuba, Ibaraki 305, Japan, email address: MAD02724@niftyserve.or.jp

${ }^{2}$ Research Engineer, Advanced Systems Group, Construction Engineering Department, Institute of Technology, Shimizu Corp., 4-17 Etchujima 3chome, Koto-ku, Tokyo 135, Japan, email address: kaku@mech.sit.shimz.co.jp

${ }^{3}$ Research Engineer, Technical Research Institute, Hazama Corp., 515-1 Nishi-mukai Karima, Tsukuba, Ibaraki 305, Japan, email address: MHE02446@niftyserve.or.jp

${ }^{4}$ Associate Researcher, System Science Institute, Waseda University, 1-21-1 Nishi-waseda, Shinjuku-ku, Tokyo 169, Japan, (Professor, School of Business Administration, Aoyama Gakuin University, 44-25 Shibuya, Shibuya-ku, Tokyo 105, Japan), email address: ytamaki@busi.aoyama.ac.jp
} 
Work package 2:

Fundamental studies on automated construction systems such as: technology of heavy material transportation, 3D position detection system, development of a theory of construction material handling process and mechanized structure realization techniques, and development of a number of new forms of material handling devices based on active and coordinated robot action system.

\section{Work package 3 :}

Study on the application of agent theory to construction management in IF7. The work package 3 has the three tasks as follows:

Task 1: Development of virtual construction dynamic simulation systems integrated with an intelligent planning, scheduling and performance management systems; This task aims at developing virtual reality based techniques for design and evaluation of construction process and producing significantly improved methodologies for field work decision management; The virtual construction dynamic simulation systems being developed will be utilized as a testbed for experimenting on the behavior of agents being developed in the task 3 ;

Task 2: Study on field factory transparency, where detailed information, proactive advises and guidelines in all locations are accessible electronically by workers for thinking and decision-making. This study includes up-to-the-minute field intelligence gathering systems and information management systems to help site-personnel better manage construction progress; and

Task 3: Study on the application of agent theory to safety management, resources management, facility management and large-scale distributed information management.

This paper presents on-going research activities of the working package 3 .

\section{2: Concept of Computerized Agents}

Construction environment is constantly changed. In fact, several changes in plan and schedule might occur by a variety of circumstances around or in construction sites. There is an opening today where there was a level floor yesterday. A great variety and volume of information often go beyond an individual's experience and ability to digest. Often necessary is reasoning based on uncertain, incomplete and sometimes inaccurate information, and then workers have to make decisions according to the degrees of partial understanding. If knowledge is specialized in a certain domain, it could be easily answered by questions concerned with that domain; otherwise, it might be too difficult to answer and so it would be necessary to do homework to seek an answer. Of necessities here are the four supports for problem-solving as follows:

(1) Computational support to perform computational tasks for workers without requiring the direct attention;

(2) Procedure-knowledge support to:

- work as teachers or consultants of novices in many activities such as diagnosis, design, monitoring, or controlling;

- provide better presentation and interpretation of safety operational standards that produces a more homogeneous treatment of established rules or procedures; and

- recovery actions that serve to lesson or negate a potential error;

(3) Thinking and decision-making support to:

- provide workers with opportunities to recognize potential hazards, develop proactive countermeasures and start monitoring; and

- pick a set of building block of information at the right level of abstraction and stimulate or guide worker's creative thinking, i.e., indication or hint; and

(4) Communication support to:

- save or share information and mail back and forth to each other; and

- help workers structure conversation and keep track of tasks.

In order to increase the efficacy of the above four supports, this study focuses on the development of computerized agents who interact with workers. The term "agent" means someone acting on behalf of someone else. An computerized agent is an entity who has own mental state being composed of beliefs, capabilities and commitments [1]. Beliefs refer to the state of the world in the past, present, or future (predicted), and to the mental state of other computerized agents. Capabilities is taken to be a primitive notion defined in terms of future-branching structures. Commitment rules are activated based on certain patterns in the incoming message and current mental condition, that is, the mental state of this computerized agent. When the time comes to execute the action, the mental state at the time will be examined to see whether the mental condition is satisfied. Generally, computerized agents may be characterized in terms of autonomous, intelligent, communication, and anthropomorphic.

In this study, agents are categorized into network agent and cyber-agent. The network agent will work on the network among large-scale distributed applications to help workers structure conversation and keep track of tasks. The cyber-agent is a virtual engineer or line-manager who inhabit within a cyberspace composed of computer systems. The cyber-agent will passively or actively walk through the cyberspace to help workers explore and capture critical factors latent in a large amount of information that may go 
into making decisions. The term "passively" means giving responses when being asked something. The term "actively" means taking actions with advice or suggestions before being asked. Although idea generation, selecting an alternative and the physical realization may still leave open questions to workers, the cyber-agent will reduce the cognitive distance between workers and cyberspace. The interaction issues between the cyber-agent and workers in this study include 1) design computer interface to match worker's cognitive processes; 2) making computer system easy to learn and use; and 3) effectively combining worker's intuitive ability with the logical function of a computer in a cooperative system.

\section{3: Network agents}

As shown in Figure 3.1, the construction management for automated sites covers wide areas: process planning and control, resources planning and control, facility management, quality control, safety management, and cost management. As the first framework of the IF7 project, the work package 3 mainly deals with the following three management categories:

1) task planing, operational instructions, and production progress control included in the "process planning and control",

2) resource requirements planning, material and machinery inventory control in site warehouses and work-in-process (WIP) in the "resources planning", and

3) safety analysis, monitoring and warming system, error recovery in the "safety management".

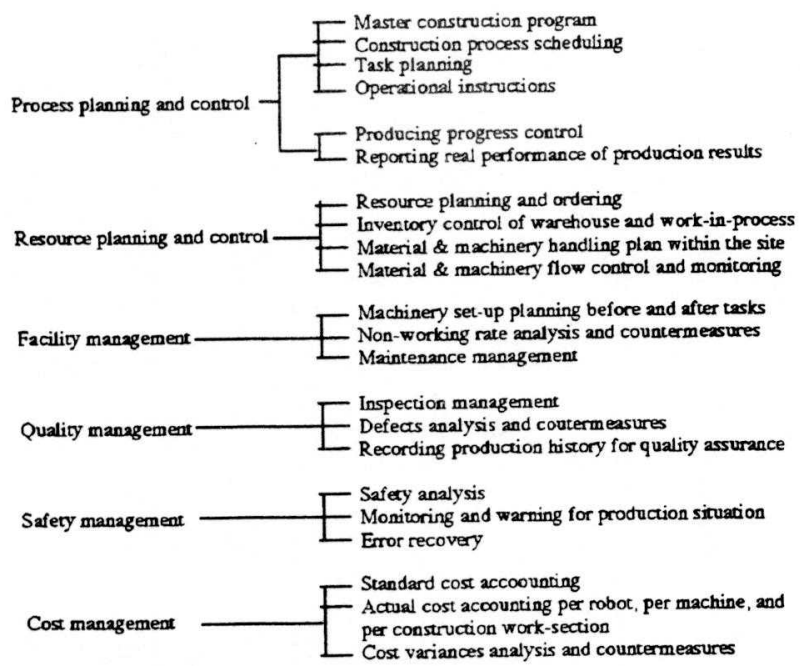

Figure 3.1: Management Categories for Work Package 3

Case study 1: Network agents in distributed applications and data
This study focuses on network agents working in or between applications and data distributed in an automated site to construct a floor ofunit boxes as shown in Figure 3.2.

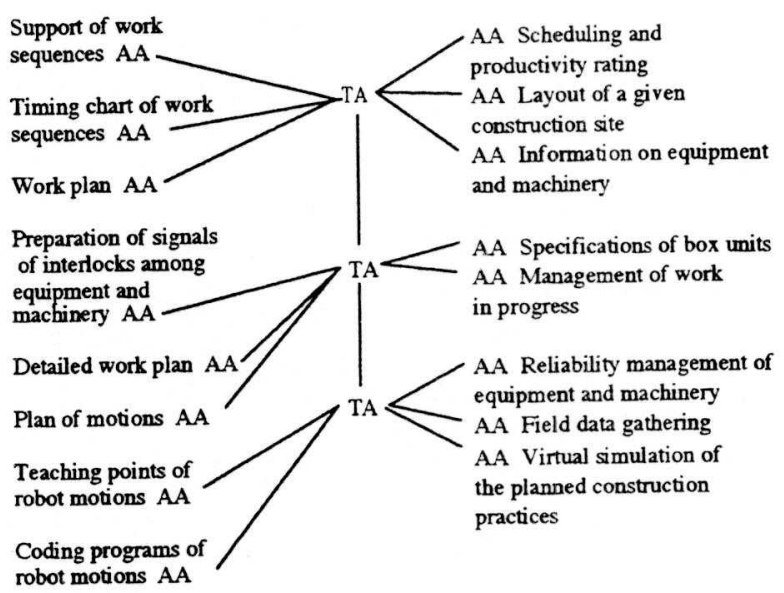

Legend: TA: transfer agent AA: application agent

Figure 3.2: Network agents in distributed applications and data

The network agents are categorized into application agent (AA) and transfer agent (TA). The application agent refers to cyber-agent as described later. The transfer agent functions to link various objects within a large-scale distributed network in a virtual lattice of applications and data In this study, an off-the-shelf soffware, system nu [nju:], is utilized as soffware platform to develop the network agents. The system nu is developed by Nihon Unisys, Ltd.. The system nu is a common object request broker architecture (CORBA) compliant product for largescale distributed applications.

\section{4: Challenges in Cyber Construction Management (C2M)}

\section{1: Virtual Dynamic Construction Simulation System}

Research and development activities of advanced computerized construction management system being conducted in Hazama Corporation is called cyber construction management (C2M). Hazama Corporation is one of general contractors in Japan. A virtual dynamic simulation construction system being developed in C2M is shown in Figure 4.1.1. Envision, which is an interactive real time 3D simulation system developed by Deneb Robotics, Inc., makes it possible forus to simulate planned construction operations. 


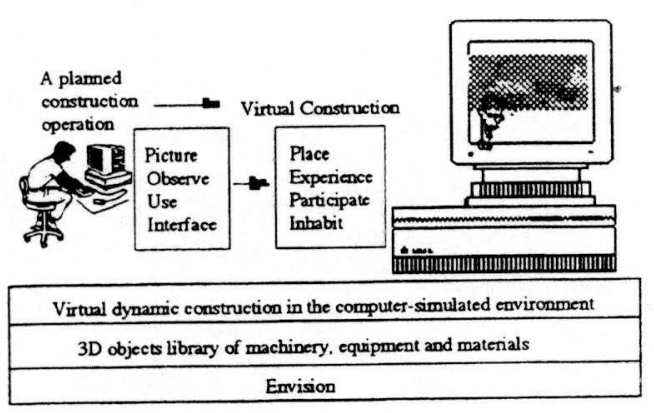

Figure 4.1.1: Virtual Construction Dynamic Simulation System in C2M

Although civil or building engineers make every attempt to address all of potential problems in their construction plan, it is often that problems do not surface until the construction operations have started. In many cases, they will build a full-scale mock-up of the product being constructed and conduct dry-run of the construction plan to identify and address those problems before the construction operations start. Since it is time consuming and expensive, we need to find more efficient and costeffective way, say, a virtual dynamic construction simulation system.

The virtual construction dynamic simulation system being developed constructs a cyberspace in which virtual construction being dynamically simulated is integrated with an intelligent planning, scheduling and performance management systems, and safety management system. It will be able to create virtual construction in 3D computersimulated environment before the planned construction is put into practice, so that we will be able to find potential hazards latent in the planning being considered and to evaluate the constructability.

\section{Case study 2: A testbed for experimenting on the behavior of cyber-agents being developed}

An assembled roof of machine room within underground power plant is built by the repetition of the sequence of the construction operations as follows: first, Each steel is brought in the underground work site; second, the each steel frame is pre-assembled at on-site yard; third, the assembled one is lifted up, transported, placed and connected to the existing roofby girders and braces; fourth, the assembled roof with wheels on rails is pushed forward at distance of $80 \mathrm{~cm}$ in one stroke operation by two hydraulic jacks; the stroke operation is subsequently repeated five times; in consequence, the assembled roof is moved forward at distance of four meters in a workshift; a sequence of these construction operations is repeated on a basis of eight-days cycle; and the repetition of the sequence results in building a roof of underground power plant. Figure 4.1.2 shows examples of virtual construction dynamic simulation of a lift-up operation of the assembled one.

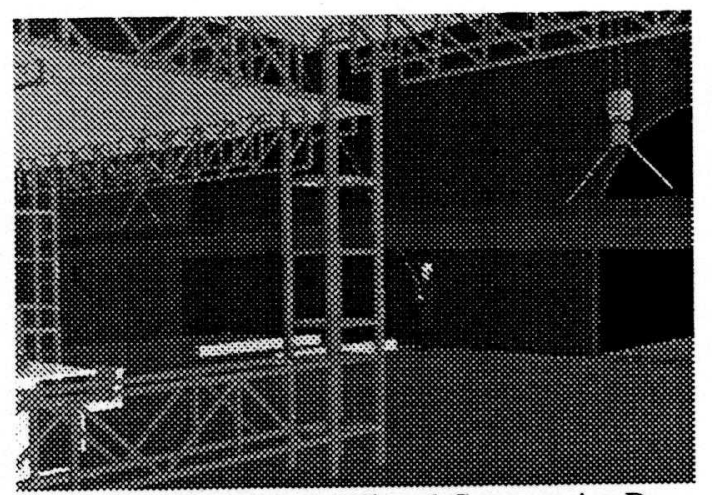

Figure 4.1.2: Examples of Virtual Construction Dynamic Simulation

Providing early visibility of a new construction operation and easily producing multiple views of the state at any stage in the construction progress demonstrates its constructability and the degrees of safety, for examples:

- showing a field of view from workers;

- confirming working space limits;

- confirming whether a behavior of a construction module is synchronized to another,

- evaluating workability from the point of view of human factor,

- walk through working space; and

- producing the detailed 2D drawings of the arbitrary cross-sections of the product being constructed.

The virtual construction dynamic simulation system is extended as a testbed for experimenting on the behavior of cyber-agents being developed. The schematic view of the testbed is shown diagrammatically in Figure 4.1.3.

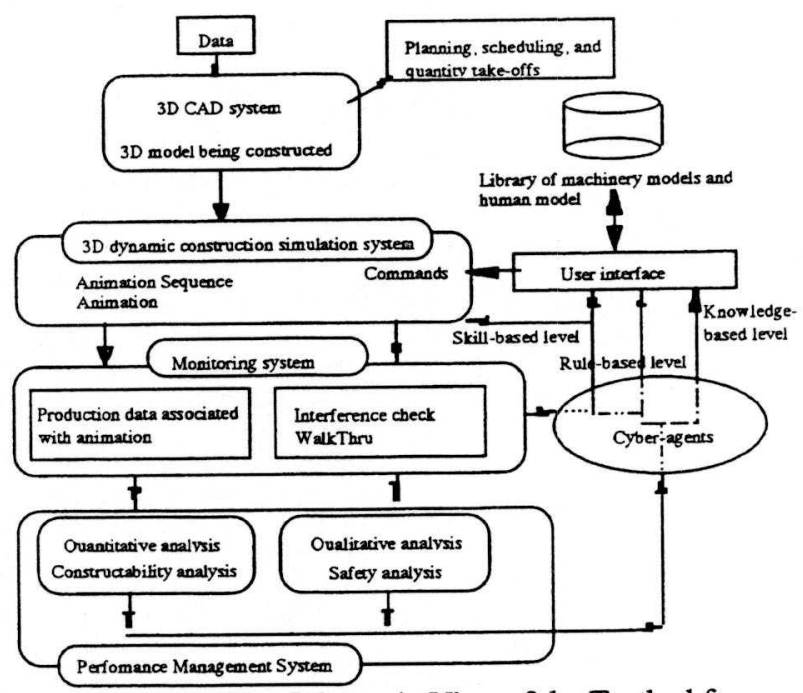

Figure 4.1.3: The Schematic View of the Testbed for Experimenting on the Behavior of Cyber-agents 


\subsection{Agentification}

Agentification in this study is proceeded by the strategy of stepwise refinement, that is, first sketching the algorithm of computerized agent in informative statements that are sufficiently clear to understand the intent, then in a mixture of some pseudo-computer-language and less formal English statements, and gradually refining until it becomes an executable code [2]. This section presents an on-going effort toward agentification in this study.

Cyber-agents within $\mathrm{C} 2 \mathrm{M}$ also have their own mental state composed of beliefs, capabilities and commitments. How can we build them in a particular given computerized system? Agentification refers to bridging the gap between the lower-level machine process and the intentional level of agent programs [1]. This section presents two case studies of agentification in the control, monitoring and warning computerized system for a semi-automated sliding system of assembled roof. The control, monitoring and warning computerized system here is functioning to: 1) monitor the behavior of the assembled roof being slid, 2) check whether its behavior is approaching its safety operating limits under operations, and 3) automatically stop its behavior in emergency.

\section{Case Study 3: Control and recovery aid}

Cyber-agent translates the raw sensory data associated with the key variables into control or error signals, signs for displaying key warning and taking proactive actions, and symbols for reasoning the underlying causes in order to invoke error recovery functions at skill-, rule- and knowledge-based levels. Figure 4.2.1 presents a schematic view of updating mental state of cyber-agent in the case of operating a hydraulic jack.

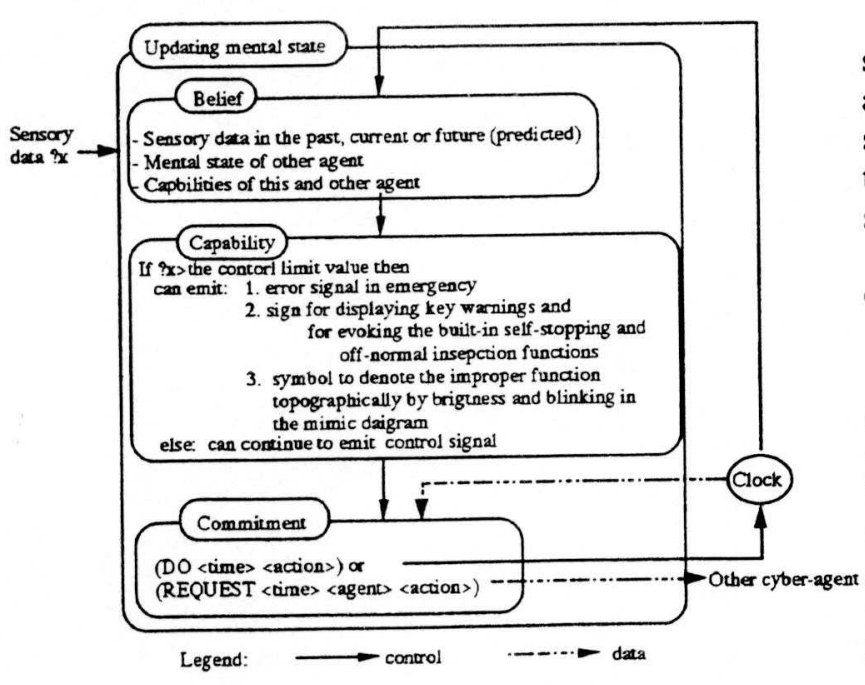

Figure 4.2.1: Updating Mental State
Signal is continuous quantitative indicator of the time-space behavior of the semi-automatic sliding system. The signal is categorized into control signal and error signal [3]. The control signal shows the transient state of behavior at a specific point in time. The error signal represents the differences between the actual state and the intended or planned state in a time-space behavior.

Cyber-agent stores a notion of the boundaries of acceptable performance ( e.g., safety control limits ) and the obligation to activate emergency shut down function if values of signal go beyond safety control limits in the capability. Workers can understand the existing state of the system by the control signal and off-normal state by the error signal.

Signs are labeled by names of states or situations of the semi-automatic sliding system [3]. Signs, which are build in IF-THEN rules, are used to select or activate stored predetermined actions that control the sequence of routine operations. Cyber-agent can display the key warnings in emergency and evoke built-in self-stopping and off-normal inspection functions when the raw sensory data goes beyond the control limit value. Workers can select and activate predetermined recovery actions by signs.

Symbols referto concepts tied to functional properties and can be used for reasoning and computation by means of a suitable representation of such properties [3]. Since workers' attention are likely to be directed at a small part of the total problem space at any one time, mimic diagram is relevant for them to direct their attention to logically important aspects of the problem space. Information is treated as symbols in the mimic diagram to topographically investigate improper function and to symptomatically reason its underlying causes. Cyber-agent here can denote the improper function topographically by making its location brightness and blinking in the mimic diagram. Workers can reason some potential faults based on symbols, as confirming and asking for additional data.

Cyber-agents in this case study provides proceduresupported knowledge to support functional understanding and represents cues for actions not only readily interpretable signs, but also the preconditions for their validity to reduce the possibility of falling into procedural traps, e.g., lapse, slip or mistake.

\section{Case Study 4: Fault warning}

Symbols in this case study are treated as abstract constructs related to the average, the standard deviation, the scale parameter and the characteristic largest value of the Type 1 extreme value distribution corresponding to the maximum values of the raw sensory data.

An engineer at the site-office intermittently analyzes the observed data, and find the suitable values of control limits and input them into the control, monitoring and warning computerized system. From the practical view point, it is very important to find values of control limits of 
the load to the two hydraulic jacks. The lower values of the control limits will frequently invoke the built-in selfstopping function and result in the poor constructability On the contrary, the higher values inevitably allow excessive loads to damage the structural elements of the assembled roof.

Figure 4.2.2 shows a schematic view of updating mental state in this case.

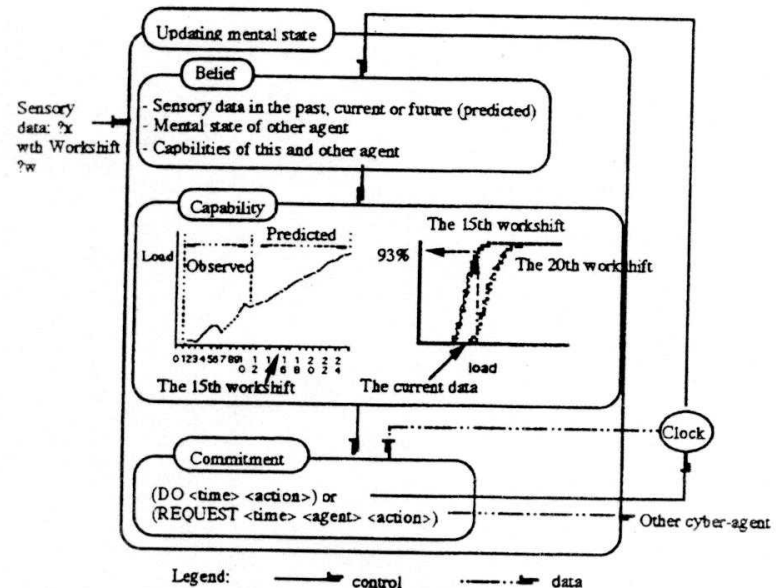

Figure 4.2.2: Updating Mental State

In the belief of cyber-agent, stored are the past, current and future (predicted) values associated with the average, standard deviation, scale parameters and characteristic largest value of the Type I extreme value distribution and the fact as common sense that the more repetition of the sequence of the construction operations, the larger those values are.

Cyber-agent stores it in her/his capability to analyze the maximum values of the raw sensory data as to the load to the hydraulic jack operations in the past and current workshifts and to construct or updating the each of the Type I extreme value distribution corresponding to the remaining workshifts. In addition, cyber-agent can dispatch evocative messages, for examples, emergency, danger, caution, in the mimic diagram when the maximum values of the current observed sensory data go beyond the value of load at the point of $90 \%$ probability of the Type I extreme value distribution (being previously constructed) corresponding to the given workshift. The evocative messages are of use to remind site-manager and engineers of some necessities to investigate potential hazard before an accident occur.

The commitment of cyber-agent send other agents, for examples, computation_support agent, of messages to summarize those past, current and future values as symbols (e.g., time plot, control chart, in the mimic diagram). Moreover, first checked are recent control influences and intervention taken by other agent when confronted with a problem, and then used are prepackaged and well-known rules or procedures. Some evocative massages are emitted when those values go beyond some threshold.

Cyber-agent in this case study provides workers with opportunities to recognize potential hazards, develop proactive countermeasures and start monitoring, so that site manager, engineers and shift supervisor can understand the interpretation of data and possible courses of construction operations.

\section{5: Further Research Program}

Agent is as yet not computerized at this point in this study. Agentification in this study starts with simple examples and proceeds by increment development. At the next stage, the algorithm of computerized agents discussed in this study will be sketched in more informative statements and in a mixture of some pseudo-computerlanguage and less formal English statements. Some of the agents will be coded to experiment their workability within a cyberspace composed of the virtual construction dynamic simulation system and other management systems.

The each task in the work package 3 is scheduled as shown in Table 5.1 .

Table 5.1: Schedule of Research Activities

$\begin{array}{lllll}\text { yy } & \text { '97 } & ' 98 & 199 & 100 \\ \text { mm } & 04 & 04 & 04 & 03 \\ \text { Task 1 } & & & & \\ \text { Task 2 } & & & & \\ \text { Task 3 } & & & \end{array}$

\section{References}

[1] Shoham, Y. (1993). "Agent-oriented programming," Artificial Intelligence, Vol. 60, pp. 51-92.

[2] Aho, A. V., Hopcroft, J. E. and Ullman, J. D. (1983). Data structures and algorithms, Addison-Wesley.

[3] Rasmussen, J. (1983). Skills, Rules, and Knowledge; Signals, Signs, and Symbols, and Other Distinctions in Human Performance Models, IEEETransactions on Systems, Man, and Cybernetics, Vol. SMC-13, No. 3, 257-266. 